\title{
A guide to intellectual disability psychiatry assessments in the community
}

\author{
Bennett Eng, Paul Addison \& Howard Ring
}

\begin{abstract}
SUMMARY
Psychiatric assessment in the community is an important part of both the initial assessment process and delivery of follow-up care in adult intellectual disability services in the UK. This article examines how such assessments can be carried out safely and explores the clinical skills required to perform them effectively. Use of the psychiatric interview and mental state examination to elicit information is discussed. Communication difficulties experienced by people with intellectual disabilities and strategies that may be employed to address these in the assessment process are also explored. The article is directed at psychiatrists, in particular specialty trainees, and other healthcare professionals who work with intellectually disabled people.
\end{abstract}

\section{DECLARATION OF INTEREST}

None.

Intellectual disability affects between 1.5 and $2 \%$ of the population in Western countries (Mefford 2012), and the prevalence of psychiatric disorders among people with intellectual disability is 4 to 5 times higher than in the general population (Rush 2004). To identify and meet the mental health needs of people with intellectual disabilities, psychiatrists and other professionals must carry out assessments either in the community or in healthcare facilities such as hospitals.

In the UK, it is increasingly common for people with intellectual disabilities to be assessed in a wide range of community settings, including nursing homes, residential homes, and special residential schools, day centres and colleges for people with disabilities. However, community intellectual disability psychiatric assessments are not widely carried out in many other countries, including Canada, Australia and the USA (Bradley 2005). In this article, we explore how psychiatric assessments of adults with intellectual disabilities are conducted in the community in the UK and describe the essential components of a comprehensive assessment. We focus on the content and process of assessment, but do not explore treatment or care in any depth.

\section{Pros and cons of community assessment}

\section{Logistic benefits}

There are many recognised benefits of a home assessment, including higher rates of patient satisfaction (Jones 1987). People with intellectual disabilities are more likely to have comorbid physical disabilities and limited mobility (Cleaver 2009), making travel to a clinic or hospital for assessment difficult. The problem of finding suitable transport and escorts for individuals who present with severe challenging behaviours is another reason why community visits are done. The opportunity for community assessments is also likely to increase the chance of successfully meeting with patients who tend not to attend appointments in clinics or hospitals. The benefits of assessing the mental health of patients in the community are well recognised in other branches of psychiatry ( $\mathrm{Ng} 2012)$.

\section{Clinical benefits}

In addition to these logistic benefits, important clinical benefits accrue from assessing a person with an intellectual disability in their own environment. In our experience, it is often possible to gain a better understanding of the person in a setting familiar to them. Problems missed in the artificial environment of a clinic or hospital may come to light when the patient, carers and family are more at ease, 'natural' and forthcoming in familiar surroundings. In a home visit, the assessor has the opportunity to see first-hand how the patient functions in their everyday context. Observation of simple tasks such as making a cup of tea can reveal whether a person's functioning has declined, possibly indicating onset of a mental or physical illness. Community assessments also help inform assessments of patient-carer interactions and of carers' needs (Scanameo 1995), ensuring
Bennett Eng is a consultant intellectual disability psychiatrist in north Norfolk and honorary senior lecturer at the University of East Anglia. His interests include medical education and the history of medicine. Paul Addison is a consultant intellectual disability psychiatrist in west Norfolk and clinical lead for community intellectual disability psychiatry services in Norfolk. Howard Ring is a university lecturer in developmental psychiatry at the University of Cambridge and an honorary consultant intellectual disability psychiatrist with Cambridgeshire and Peterborough NHS Foundation Trust. Correspondence Dr Bennett Eng, Willowbank, Little Plumstead Hospital, Norwich NR13 5EW, UK. Email: b.eng@nhs.net 
that appropriate support, such as respite or home help, can be provided to carers without delay. In addition, these assessments enable the assessor to obtain a collateral history from people who work with the patient but who would not accompany them to a clinic.

\section{Disadvantages}

Although there are many recognised benefits to home and community assessments, there are several disadvantages that should be considered. Patients and carers may be uncomfortable with visitors coming to their home, and access to some homes may not be easy, owing to lack of parking or for other reasons. Absence of equipment to carry out a physical examination, if that were deemed necessary, is another potential problem. Other disadvantages include more travel time for busy healthcare professionals and possible health and safety risks, including vulnerability of unaccompanied staff to allegations of misconduct or to assault by patients or others.

\section{Preparation for initial community assessment}

\section{Referral}

All intellectual disability services in the UK accept referrals from general practitioners (GPs). Some also accept referrals from other sources, such as patients, their carers (including family or paid support workers) or social workers. The GP has an important role in the referral process. As the primary healthcare provider, the GP has a central position in managing comorbid physical illness so, regardless of where the referral originates, good communication with the GP is essential. The GP frequently has a long-standing relationship with the patient and can provide useful information and insights into their mental state and physical health.

Gathering information from the referral letter or referral form is an important task. This information is used in provisional differential diagnosis and in identifying likely healthcare needs and communication difficulties. With this information, a visit with the right mix of professionals can be planned. For example, if a referral letter points out that a person requires assistance with communication in addition to a mental health assessment, a joint visit of a psychiatrist and speech and language therapist might be appropriate. Information from the referral document is also used to identify any risks in carrying out a community assessment and the urgency of the required response.

\section{Planning the first visit}

The first visit to a patient in the community needs to be planned carefully. We believe that it would be ideal for initial assessments to be discussed with the multidisciplinary team meeting before the visit. Matters that need to be discussed include the number of professionals needed for the visit, the skill mix best suited to the particular assessment and safety measures to be taken. Safety of healthcare professionals carrying out home visits must be carefully considered (Campion 1997). Safety measures might include panic alarms, mobile phones and, in extreme circumstances, request for police escort or assistance.

Before the initial assessment, telephone contact or formal written communication to arrange the visit should be established. A member of the team or the secretary should ask about availability of parking and safety issues such as dangerous dogs. The need for carers or family members who know the patient well to be present to enable a collateral history to be taken should also be made clear to the carers and patient.

\section{The assessment}

\section{Visiting the patient}

When visiting a patient in the community, the appearance of the neighbourhood will often give some indication of safety. Before entering a private home, information can be gathered by observing the state of the building and surrounding environment.

After introductions have been made, the psychiatric interview begins with identification of a suitable interview room. In residential homes, day centres, special schools or colleges, a quiet room or specially designated interview room is usually available if members of staff of these centres have been given prior notice that an assessment is due to take place.

For assessments in private homes where patients stay with their parents or others who are their main carers, the patient and carers should be asked where they would like the interview to be carried out. The patient may indicate a preference for the room that they are most comfortable in, such as the kitchen. The wishes of the patient or carer should be respected if there are no risk concerns. If risk concerns are present, assessments should be carried out near exits to enable easy escape.

\section{The psychiatric interview}

The psychiatric interview of an intellectually disabled patient includes a comprehensive psychiatric history resembling that taken from a 
person without intellectual disability. In addition to a full psychiatric history, particular attention needs to be paid to the patient's developmental, educational and, if relevant, employment history. A summary of the information that should be elicited in a comprehensive initial interview is laid out in Box 1, and some areas where special attention is required are detailed below.

\section{Autism spectrum disorders}

The interviewer should consider whether the patient has features of an autism spectrum disorder, as about $75 \%$ of people with autism have intellectual disability requiring substantial social and educational support (Mefford 2012). Features of autism spectrum disorders include impaired communication, impaired social interaction, restricted interests and repetitive behaviours (World Health Organization 1992).

\section{Challenging behaviour}

It is essential to ask whether the patient currently presents challenging behaviours such as hitting out, or has a history of such behaviours. These are not uncommon in intellectually disabled people, with prevalence studies reporting rates between 5.7 and 14\% (Xeniditis 2001). Possible triggers, together with the effects of and dangers posed by such behaviours, should be considered during the interview. Before considering use of psychotropic medication for chronic behavioural disturbance, it is important to investigate underlying physical or psychiatric illnesses and environmental factors that might be causing it (Tyrer 1997). Suitable enquiries should therefore be made during the interview.

\section{Self-injurious behaviours}

Similarly, self-injurious behaviours such as headbanging and biting should be subject to detailed enquiry during the psychiatric interview. Such behaviours may be relatively frequent and can have serious effects on the patient's health. An epidemiological study in the UK showed that selfinjurious behaviours were present in $17.4 \%$ of the intellectually disabled population (Collacott 1998), with prevalence increasing as IQ score falls. These behaviours are best understood as being caused by a combination of organic and environmental factors (Deb 1998; Mikhail 2001). Therefore, a detailed enquiry into any possible causes and triggers should be made during the interview.

\section{Epilepsy and seizure}

Enquiry about whether the person has epilepsy or a history of epileptic seizures is also necessary.
BOX 1 Information to be elicited in the initial psychiatric interview of an individual with an intellectual disability
- Presenting complaint

- History of presenting complaint (a detailed account of presenting problems, including the ability of carers to cope)

- Medical history (especially of seizures, epilepsy, constipation, infection, visual or hearing problems; presence of a recognisable genetic syndrome, if known)

- Psychiatric history (including all previous contact with psychiatric, intellectual disability, psychology or educational psychology services; history of autism spectrum disorders, challenging behaviours, self-injurious behaviours)

- Family history (including a family tree and family history of intellectual disability, mental and physical health problems, and genetic syndromes)
- Developmental history, starting from the mother's pregnancy (problems during pregnancy/delivery/postnatal period) problems or delays in development, presence of any features of autism, current level of functioning and ability to carry out the activities of daily living (ADL), results of formal 10 testing, if available)

- Educational history la list of all educational institutions attended and whether they were special or mainstream schools; educational attainment, challenging behaviours were displayed in these institutions and the nature of any such behaviours)

- Employment history (all periods of paid sheltered or voluntary employment)

- Forensic history

- Alcohol and drug history whether the person was bullied, whether

This is because epilepsy is very common in people with intellectual disability, affecting roughly 1 in 5 intellectually disabled adults (Joint Epilepsy Council 2005). Epilepsy can have a major impact on the patient's mental health. Depressive symptoms occur in up to $60 \%$ of people with intractable epilepsy (Lambert 1999), and the prevalence of psychotic illness in people with epilepsy is at least $4 \%$ (Blumer 2000). Anti-epileptic drugs have also been associated with new-onset depression and psychosis (Harden 2002). Consequently, a detailed understanding of possible triggers for seizure activity, the type of seizures the patient has, the treatment they are receiving and the severity of the condition is important.

\section{Observation of behaviour and current mental state}

As part of the psychiatric interview, observation of the person and assessment of their mental state are essential. Particular attention needs to be paid to their appearance and behaviours. These may be indicative of an underlying genetic syndrome, as well as of mental states that limited communication skills might otherwise make difficult to diagnose. Individuals with more severe intellectual disabilities are more likely to present with psychomotor agitation, irritability and disturbed behaviour when depressed (Prasher 1999). Box 2 lays out the components of the mental state examination for an intellectually disabled person. 
BOX 2 Mental state examination for someone with an intellectual disability

- Appearance (including hygiene standards and other evidence of possible neglect, such as weight, hydration and state of clothing)

- Behaviour (including self-harming and challenging behaviours, motor restlessness, involuntary movements, tremors)

- Mood

- Speech (including repetitive or stereotyped patterns of speech, poverty/lack of speech or limited nature of vocabulary and understanding, screaming or shouting)

- Thoughts

- Perception

- Insight

- Cognition (including an estimate of level of intellectual disability from the person's functional abilities if formal I0 testing has not been carried out)

Assessment tools such as the Autism Diagnostic Observation Schedule (ADOS; Lord 1989), the Diagnostic Interview for Social and Communication Disorders (DISCO; Wing 2002) and the Dementia Questionnaire for People with Learning Disabilities (DLD; Eurlings 2006) can be used to support the assessment, but such tools are not a substitute for a comprehensive psychiatric interview.

Two case vignettes illustrating common clinical presentations in intellectual disability psychiatry are featured in Boxes 3 and 4. The vignettes are entirely fictional, but they are representative of the patients seen by community intellectual disability psychiatrists.

\section{Physical examination}

The assessing psychiatrist should conduct a physical examination if they suspect that the patient has physical health problems, such as a chest infection, which have not been reported to the GP. Although the GP, as the primary care physician, is responsible for treating physical health problems, the intellectual disability psychiatrist, together with other health professionals, can play an important role in alerting the GP and acute hospitals or medical services to the physical health needs of intellectually disabled patients (Royal College of Psychiatrists 2011).

It is very important to note that underlying physical illness is often a trigger of challenging behaviours or a deterioration in mental health (De Winter 2011). If the psychiatrist suspects that underlying physical causes may be contributing to
B0X 3 Challenging behaviours

A 21-year-old woman with a moderate intellectual disability, living in a residential home with four other residents, was referred to the mental health services by her GP because she had been throwing objects and hitting out at her carers. She had also been banging her head against the wall. She appeared agitated and restless during the home assessment. A collateral history from her carers in the residential home revealed that she had been incontinent of urine over the past 2 weeks. During the home assessment, she had to go to the toilet several times to pass urine. Using a urine dipstick test, a urinary tract infection was diagnosed. After the woman had completed a course of oral antibiotics, her challenging behaviours stopped.

a person's deteriorating mental state, behaviour or functional abilities, physical examination should be considered. In addition, a careful history of potential physical problems should be taken, including, in particular, issues such as constipation and gastro-oesophageal reflux, which are relatively common in people with intellectual disabilities. Pain or discomfort associated with physical problems can lead to a range of psychobehavioural disturbances.

A history should be obtained by talking to well-informed carers, but it may not be easy to carry out a physical examination if the patient is unwilling to cooperate. If this is the case, the patient and their carer should be informed that a physical examination is needed and given an explanation as to why it may be helpful. The GP must also be informed.

Features to look out for in the physical examination of intellectually disabled patients are

BOX 4 Presenile dementia in Down syndrome

A 46-year-old man with Down syndrome, living with his elderly parents (who are his carers), was referred to a GP by his parents, who were concerned that he was not attending to his personal hygiene and other chores as well as he used to. The GP referred him to mental health services with concerns that he may be confused. No psychotic or depressive symptoms were elicited on mental state examination. The collateral history from the man's parents revealed that his functional abilities had begun to deteriorate several months ago, with insidious onset. A home assessment revealed that his bedroom was untidy but the rest of the house was well kept. In the course of the assessment, the man became upset when his parents asked him to make a pot of tea, as he had forgotten where the kettle and tea bags were kept. 
listed in Box 5. The assessing psychiatrist should note features suggesting a genetic syndrome associated with intellectual disability and consider referral to the clinical genetics service for a definitive diagnosis, if appropriate. The features of several genetic syndromes associated with intellectual disability are laid out in Box 6 .

\section{Risk assessment}

Carrying out a comprehensive risk assessment is an essential component of any psychiatric assessment (Dixon 2007). Risk assessment encompasses risks to the patient and risks posed by the patient to others or to property. In addition to the normal risk assessment undertaken in any psychiatric assessment, the psychiatrist must also be aware of the dangers posed by self-neglect, self-injurious behaviours such as biting and headbanging and other challenging behaviours when assessing a person with intellectual disabilities. Observation of the patient's behaviour during the assessment, a detailed collateral history from carers, physical examination of the patient to assess the extent of injuries, and inspection of any damage to the home or property caused by the patient's behaviour can help inform the assessor of the severity of the risks posed by a patient's challenging behaviours.

Importantly, risk assessment of people with intellectual disabilities should include consideration of their vulnerability to abuse by others.

In drawing up a comprehensive risk assessment, it is essential that the views of, and input from, other members of the multidisciplinary team are considered and incorporated as appropriate.

In any risk assessment, consideration should be given to whether the risks posed by a person can be safely managed in a community setting. Admission to an in-patient unit for further assessment and treatment should be considered if the risks identified cannot be safely managed in the less restrictive environment of a community placement. Recourse to mental health legislation to facilitate admission may be appropriate in such circumstances.

\section{Effective communication in the assessment}

People with intellectual disabilities frequently have problems communicating their needs to doctors (Wullink 2009). They may have sensory deficits, difficulty articulating words and limited ability to understand and use appropriate language. It is important for doctors to be aware of such communication difficulties and of strategies, tools and approaches that may be used to aid communication so that an effective assessment can take place.
BOX 5 Features to look for in the physical examination of a person with an intellectual disability

- Neurological deficits

- Low muscle tone

- Cardiac murmurs

- High blood pressure

- Evidence of infection, especially chest and urinary tract infections

- Pressure sores

- Bruising or other injuries sustained from falls or selfinjurious behaviours

- Hard, lumpy abdomen, possibly indicating constipation

- Evidence of pain on limb movement, suggesting possible injury or joint problems

- Signs suggestive of possible physical abuse, including choke marks around the neck, human bite marks, cigarette burns, unusual fractures

\section{Communication strategies and aids}

Strategies to aid effective communication include talking slowly, asking the person whether they understand what is being said and asking them to repeat what has just been explained to them to check their understanding. The presence of a speech and language therapist during an assessment can help.

Box 7 lists a few tools and approaches that can aid communication with intellectually disabled people. In Makaton, signs and symbols are used simultaneously with speech to support spoken language. When using a 'talking mat', the doctor and patient can place picture symbols

BOX 6 Features of genetic syndromes associated with intellectual disability

\begin{tabular}{ll}
\hline Down syndrome & $\begin{array}{l}\text { Single palmar crease, oblique } \\
\text { eye fissures, poor muscle tone, } \\
\text { protruding tongue, Brushfield } \\
\text { spots (white spots on iris) }\end{array}$ \\
Fragile-X syndrome & $\begin{array}{l}\text { Elongated face, large ears, } \\
\text { large testes, high arched palate, } \\
\text { hyperextensible joints, poor } \\
\text { muscle tone, hyperactivity }\end{array}$ \\
Prader-Willi syndrome & $\begin{array}{l}\text { Obesity, prominent nasal bridge, } \\
\text { compulsive behaviour, skin } \\
\text { picking, extreme appetite } \\
\text { 'Elfin' facial features, cheerful } \\
\text { demeanour, poor concentration, } \\
\text { disinhibition, hyperactivity }\end{array}$
\end{tabular}


BOX 7 A few communication aids used by people with intellectual disability

- Signing, including Makaton and British Sign Language

- Symbols, photos, talking mats

- Picture exchange communication systems (PECS), including communication books and communication boards

- Voice output communication devices

on the mat to facilitate communication. 'Picture exchange communication systems' allow patients to communicate their needs by selecting an appropriate picture. Voice output communication devices produce a synthesised or digitised voice to enable a person who cannot speak or whose speech is unintelligible to communicate. For people with intellectual disabilities, these devices typically have a touchscreen showing pictures and symbols.

\section{Cognitive assessments to identify level of disability}

As part of the assessment, it is useful to establish the level of intellectual disability, as this may give some indication of the person's mental and physical health needs. If formal cognitive assessment has not previously been carried out, the psychiatrist should consider making a preliminary approximate estimate of the level of intellectual disability. The person's functional abilities and communication skills can be used as a guide in making this preliminary estimate. Box 8 lays out the skills and abilities commensurate with each level of intellectual disability.

BOX 8 Guide to making a rough estimate of the level of intellectual disability based on functional abilities of the patient

$\begin{array}{lc}\begin{array}{l}\text { Mild intellectual disability } \\ \text { - Usually able to hold a conversation and } \\ \text { engage in the interview }\end{array} & \begin{array}{c}\text { Severe intellectual disability } \\ \text { - Marked degree of motor impairment and } \\ \text { other deficits }\end{array} \\ \text { - Most achieve independence in self-care, } & \begin{array}{c}\text { - Limited language and some may use signs } \\ \text { daily living and domestic skills }\end{array} \\ \text { - History of academic problems common but } & \text { - Epilepsy and neurological deficits common } \\ \text { most are capable of independent work } & \text { Profound intellectual disability } \\ \text { Moderate intellectual disability } & \text { - Severely limited ability to understand or } \\ \text { - Usually requires some supervision in } & \text { comply with requests } \\ \text { self-care } & \text { - Mobility problems, incontinence, epilepsy } \\ \text { - Some may have basic reading, writing or } & \text { and visual impairment common } \\ \text { counting skills } & \text { - Inability to generate most intentional } \\ \text { - Able to do simple tasks with supervision } & \text { actions } \\ \text { and can engage in social activities } & \end{array}$

Ideally, this rough estimate should be followed up by a formal IQ assessment using a standardised tool such as the Wechsler Adult Intelligence Scale (WAIS; Wechsler 2010) if a trained assessor is available in the clinical team. The WAIS is by far the most widely used IQ test in the UK, where formal IQ assessments are usually undertaken by educational or clinical psychologists. Such assessments will not always be possible, given the limited resources available to many clinical teams in the UK.

\section{Follow-up assessments of known patients}

Patients who have already been assessed by psychiatric services may need to be followed up with further assessments in the community. In organising these assessments, the psychiatrist will already have a significant amount of information about the patient and their environment, including information on whether there are any safety concerns or risks to the psychiatrist going out to do the assessment. Follow-up assessments require the psychiatrist to focus on the patient's presenting needs and any changes in their mental state and environment since the last assessment. By carefully considering any changes and comparing them with the person's history, a good understanding of the person and any problems they may have can be achieved. In the course of a follow-up assessment, the necessity of further visits and when they should take place should also be discussed with the patient and carers.

\section{Drawing up a treatment plan after assessment}

Drawing up a treatment plan after gathering the necessary information in an assessment involves making a diagnosis, identifying risks to the patient, risks posed by the patient and the patient's unmet needs. Although diagnostic classification clearly provides guidance on morbidity, mental health services are increasingly adopting a biopsychosocial approach to assessment and treatment of mental health problems in people with intellectual disabilities (Costello 2006). The aim of this approach is to gain a comprehensive understanding of the biological, psychological and social causes of the person's mental health problems. With a better understanding of these factors in a particular person, approaches to treatment that address them can be better identified.

The biopsychosocial approach to formulation has now been widely adopted by mental health services in the UK, but it can be augmented 
by other frameworks, including systemic and psychodynamic formulations. In the development of a comprehensive treatment plan, suitable consideration should be given to whether the person might benefit from psychological treatments and is willing to engage with a therapist in individual sessions or participate in group sessions.

When suitable treatment approaches have been identified, the patient's capacity or ability to give informed consent to any proposed intervention must be carefully considered. The person's capacity to consent should be assessed in accordance with mental health and mental capacity legislation in the particular country or jurisdiction. If necessary, use of mental health or mental capacity legislation to lawfully facilitate treatment in the patient's best interests should be considered. Psychiatrists must be familiar with the relevant legislation in their locality. In England and Wales, for example, this includes the Mental Capacity Act 2005 (Department for Constitutional Affairs 2007), the Mental Health Act 1983 (as amended in 2007) (Department of Health 2008; Welsh Assembly Government 2008) and the Deprivation of Liberty Safeguards (Ministry of Justice 2008).

A patient with complex needs may require treatment and support from a range of professionals, including speech and language therapists, physiotherapists, occupational therapists, psychologists, social workers, community nurses and the GP. If there are serious physical health problems that require urgent treatment, admission to an acute hospital should be considered as a priority. If a genetic diagnosis has not already been established, the psychiatrist, together with the patient and family, can consider whether referral to a clinical genetics service may be beneficial in addressing potential health and reproductive issues. Thus, the assessment by the psychiatrist may be only the first step in the development of a comprehensive treatment plan, as assessments by other professionals may be required to fully identify the needs of the individual and the range of treatment options available.

\section{Conclusions}

A good intellectual disability psychiatry assessment is one that not only explores the presenting problems and history of the individual. It also accurately and comprehensively identifies their needs, so that effective treatment and management plans that address these needs can be put in place. In the assessment of an intellectually disabled person, the psychiatric assessment may be only one component of a comprehensive assessment approach that identifies all the person's needs. A multidisciplinary team approach to assessment and treatment should be adopted, in particular when a patient has multiple, complex needs.

MCQ answers

$\begin{array}{lllll}1 \mathrm{~b} & 2 \mathrm{a} & 3 \mathrm{c} & 4 \mathrm{~d} & 5 \mathrm{c}\end{array}$

\section{References}

Blumer D, Wakhlu S, Montouris G, et al (2000) Treatment of the inter-ictal psychoses. Journal of Clinical Psychiatry 61: 110-22.

Bradley E, Lofchy $\mathrm{J}$ (2005) Learning disability in the accident and emergency department. Advances in Psychiatric Treatment 11: 45-57.

Campion EW (1997) Can house calls survive? New England Journal of Medicine 337: 1840-1.

Cleaver S, Hunter D, Ouellette-Kunz H (2009) Physical mobility limitations in adults with intellectual disability: a systematic review. Journal of Intellectual Disability Research 53: 93-105.

Collacott RA, Cooper SA, Branford D, et al (1998) Epidemiology of selfinjurious behaviour in adults with learning disabilities. British Journal of Psychiatry 173: 428-32.

Costello H, Bouras N (2006) Assessment of mental health problems in people with intellectual disabilities. Israel Journal of Psychiatry and Related Sciences 43: 241-51.

Deb S (1998) Self-injurious behaviour as part of genetic syndromes. British Journal of Psychiatry 172: 385-8.

Department for Constitutional Affairs (2007) Mental Capacity Act 2005 : Code of Practice. TSO (The Stationery Office).

Department of Health (2008) Code of Practice: Mental Health Act 1983. Published Pursuant to Section 118 of the Act. TSO (The Stationery Office).

De Winter CF, Jansen AAC, Evenhuis HM (2011) Physical conditions and challenging behaviour in people with intellectual disability: a systematic review. Journal of Intellectual Disability Research 55: 675-98.

Dixon M, Oyebode F (2007) Uncertainty and risk assessment. Advances in Psychiatric Treatment 13: 70-8.

Eurlings HAL, Evenhuis HM, Kengen MMF (2006) Dementia Questionnaire for People with Learning Disabilities (DLD). Pearson Assessment.

Harden CL, Goldstein MA (2002) Mood disorders in patients with epilepsy: epidemiology and management. CNS Drugs 16: 291-302.

Joint Epilepsy Council (2005) Epilepsy Prevalence, Incidence and Other Statistics. Joint Epilepsy Council.

Jones SJ, Turner RJ, Grant JE (1987) Assessing patients in their homes. Psychiatric Bulletin 11: 117-9.

Lambert MV, Robertson MM (1999) Depression in epilepsy: etiology, phenomenology and treatment. Epilepsia 40 (suppl 10): S21-47.

Lord C, Rutter M, Di Laore PC, et al (1989) Autism Diagnostic Observation Schedule (ADOS). Western Psychological Services.

Mefford HC, Batshaw ML, Hoffman EP (2012) Genomics, intellectual disability and autism. New England Journal of Medicine 366: 733-43.

Mikhail AG, King BH (2001) Self-injurious behaviour in mental retardation. Current Opinion Psychiatry 14: 457-62.

Ministry of Justice (2008) Mental Capacity Act 2005: Deprivation of Liberty Safeguards. Code of Practice to Supplement the Main Mental Capacity Act 2005 Code of Practice. TSO (The Stationery Office).

$\mathrm{Ng} \mathrm{B}$, Atkins M (2012) Home assessment in old age psychiatry: a practical guide. Advances in Psychiatric Treatment 18: 400-7.

Prasher V (1999) Presentation and management of depression in people with learning disability. Advances in Psychiatric Treatment 5: 447-54.

Royal College of Psychiatrists Faculty of Learning Disability (2011) Future Role of Psychiatrists Working with People with Learning Disability (Faculty Report FR/LD/1). Royal College of Psychiatrists.

Rush KS, Bowman LG, Eidman SL, et al (2004) Assessing psychopathology in individuals with developmental disabilities. Behaviour Modification 28 : 621-37.

Scanameo A, Fillit $\mathrm{H}$ (1995) A practical guide to seeing patients at home. Geriatrics 50: 33-7. 
Tyrer S (1997) The use of psychotropic drugs. In Seminars in the Psychiatry of Learning Disabilities (ed 0 Russell): 205-22. Royal College of Psychiatrists.

Wechsler D (2010) Wechsler Adult Intelligence Scale - Fourth UK Edition (WAIS-IV UK). Pearson Assessment.

Welsh Assembly Government (2008) Mental Health Act 1983: Code of Practice for Wales. TSO (The Stationery Office).

Wing L, Gould J (2002) The Diagnostic Interview for Social and Communicative Disorders. Centre for Social and Communication Disorders.
World Health Organization (1992) The ICD-10 Classification of Mental and Behavioural Disorders: Clinical Descriptions and Diagnostic Guidelines. WHO

Wullink M, Veldhuijzen W, Lantman-de Valk HM, et al (2009) Doctorpatient communication with people with intellectual disability: a qualitative study. BMC Family Practice 17 Dec (doi: 10.1186/1471-229610-82).

Xeniditis K, Russell A, Murphy D (2001) Management of people with challenging behaviour. Advances in Psychiatric Treatment 7: 109-16.

\section{MCOs}

Select the single best option for each question stem

1 Advantages of a home visit do not include:

a greater likelihood of obtaining a collateral history

b saving time for the psychiatrist

c more accurate assessment in the 'natural' environment

d improved appointment attendance

e greater opportunity to assess the patient's functional abilities in their home.
2 Features associated with fragile- $X$ syndrome include:

a high, arched palate

b extreme appetite

c skin picking

d 'elfin' facial features

e 'Brushfield' spots.

3 A person with moderate intellectual disability is usually able to:

a work as a school teacher

b live independently

c carry out simple tasks with supervision

$\mathrm{d}$ work as a university lecturer

e achieve full independence in self-care.
4 The proportion of people with autism who also have an intellectual disability is:
a $5 \%$
b $15 \%$
c $55 \%$
d $75 \%$
e $95 \%$.

5 An initial referral to intellectual disability psychiatry services in the UK must involve: a a speech and language therapist

b an occupational therapist

c a general practitioner

$\mathrm{d}$ a relative

e a psychologist. 